\title{
High spin polarization of optically-oriented trions in $p$-doped GaAs-AlGaAs quantum wells
}

\author{
Klaus Wagenhuber*, Vadim Shalygin ${ }^{\dagger}$, Ulrich Niedermeier*, Christian Gerl*, \\ Werner Wegscheider*, Tobias Korn*, Robert Schulz*, Christian Schüller*, \\ Eugeniyus L. Ivchenko** and Sergey Ganichev*
*Institut für Experimentelle und Angewandte Physik, Universität Regensburg, 93040 Regensburg, Germany ${ }^{\dagger}$ St. Petersburg State Polytechnical University, 195251 St. Petersburg, Russia
${ }^{* *}$ A. F. Ioffe Physico-Technical Institute, Russian Academy of Science, 194021 St. Petersburg, Russia

\begin{abstract}
We performed cw-photoluminescence (PL) measurements on a $15 \mathrm{~nm}$ wide $p$-doped $\mathrm{Al}_{0.3} \mathrm{Ga}_{0.7} \mathrm{As}-\mathrm{GaAs}-$ $\mathrm{Al}_{0.3} \mathrm{Ga}_{0.7} \mathrm{As}$ quantum well structure at low temperatures. By irradiating the sample with circularly polarized light and analyzing the degree of circular polarization of the PL signal, we found a significantly higher degree of spin polarization for charged excitons (trions) than for neutral excitons. Time-resolved pump-probe and Faraday-rotation experiments give an additional information concerning the exciton and trion resonant photoexcitation.
\end{abstract}

Keywords: spin polarization, exciton, trion, photoluminescence

PACS: 72.25.Fe, 72.25.Rb, 73.21.Fg, 73.61.Ey

Optical spin orientation of charge carriers belongs to the most important experimental means in spintronics research [1]. We have investigated the spin polarization of optically oriented neutral excitons and positivelycharged trions in a dilute two-dimensional hole gas and found an up to about four times larger polarization degree of the trions as compared to the neutral excitons.

In our experiment we use a $780 \mathrm{~nm}$ semiconductor laser and a $\lambda / 4$ plate to create circularly polarized light for excitation. The sample is a $p$-type modulation-doped (001)-grown single quantum well $\left(p=0.9 \times 10^{11} \mathrm{~cm}^{-2}\right.$, $\mu=197.000 \mathrm{~cm}^{2} / \mathrm{Vs}$ ) that is mounted on the cold finger of a He-flow cryostat. All measurements are performed at a temperature of $T=5 \mathrm{~K}$. Excitation and detection of the PL signal are performed normal to the plane of the quantum well. The degree of circular polarization of the PL signal is analyzed. For the time-resolved measurements we use a mode-locked Ti-Sa laser and a translation stage to delay the probe pulse with respect to the pump pulse. The differential transmission (DT) signal is measured on a thinned sample with the pump and the probe beam collinearly polarized. For time-resolved Faradayrotation (TRFR) measurements, the pump beam is circularly polarized and the probe beam is linearly polarized.

The PL spectrum shows two characteristic peaks which can be attributed to the recombination of neutral excitons $\left(X^{0}\right)$ and trions $\left(X^{+}\right)$. Power-dependent measurements confirm this interpretation $[2,3]$. In order to analyze the spin polarization of $X^{0}$ and $X^{+}$, we irradiate the sample with circularly polarized light and record spectra for both helicities of the PL signal (Fig. 1). De-

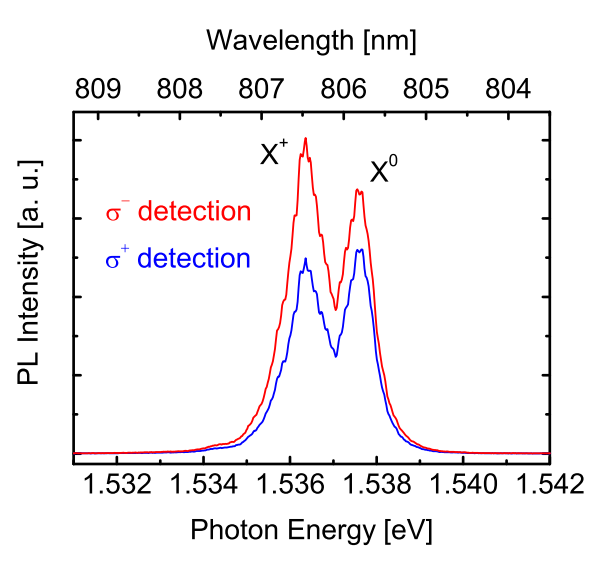

FIGURE 1. Circularly polarized PL signals $\left(\sigma^{+}\right.$and $\left.\sigma^{-}\right)$ resulting from $\sigma^{-}$-excitation.

pending on the power of excitation, the spin polarization of $X^{+}$reaches values that are almost four times larger than the values obtained for $X^{0}$. Figure 2 shows the degree of circular polarization of the PL signals for various excitation powers. As compared to $X^{+}$, the $X^{0}$ polarization is much more sensitive to the pump intensity. This can be understood taking into account the splitting of the radiative doublet of excitons localized on an anisotropic island formed by a well-width fluctuation. With increasing the intensity, the occupation of deep localized excitonic states is saturated and the shallower localized states are involved in the recombination. If the islands responsible 


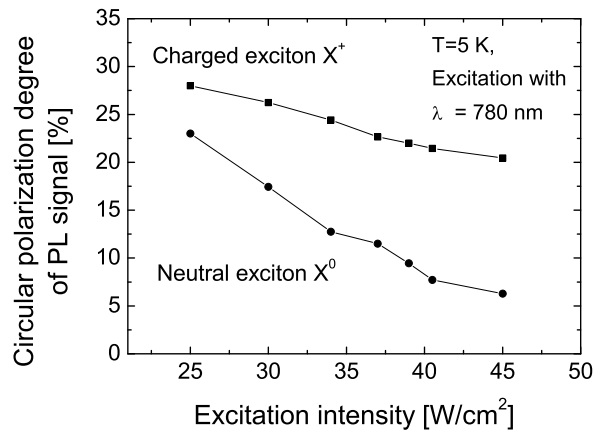

FIGURE 2. Circular polarization degree of PL signals from neutral and charged exciton recombination in an excitation density range where both peaks are present in the PL spectrum. For excitation circularly polarized light with $\lambda=780 \mathrm{~nm}$ was used.

for the shallower states are more anisotropic, the spin decoherence arising because of the anisotropic splitting is enhanced, resulting in the lower PL polarization.

The DT experiment mainly probes the transient bleaching of the optical absorption due to the creation of photocarriers. In our experiments we used 100 fs pulses with an energetic FWHM of about $20 \mathrm{meV}$, which were centered at an energy of $1.535 \mathrm{eV}$. Comparing this to the PL spectra in Fig. 1, it is obvious that we excite simultaneously $X^{+}$and $X^{0}$. In the DT experiment we observe two processes (see Fig. 3). On the ultrafast timescale, we find that a very small part of the DT signal decays within the first few picoseconds, as seen in Fig. 3b. On the slower timescale, we find a monoexponential decay with a time constant of about 890 ps, shown in Fig. 3a. The ultrafast decay can be attributed to the recombination of excitons, which are directly generated into the narrow center-of-mass momentum cone of radiative states or to free trions generated by the pulse [3]. With the measurements done so far, we can not discriminate between these two processes. This would be possible by selective resonant excitation of either the $X^{+}$or the $X^{0}$ with energetically narrower pulses, which is planned for the near future. The slow decay in Fig. 3a can be related to hot excitons, which occupy the states outside the above cone and are occasionally scattered into the cone or to a system of localized excitons and trions. The monoexponential character of the decay evidences the hot-exciton mechanism of the pump-probe response with negligible contribution from trion states. This suggests for exciton resonant photoexcitation indirect optical transitions assisted by resident heavy holes, which can carry an excess momentum.

The TRFR measurement probes the spin relaxation time of the photogenerated carriers. In our measurement

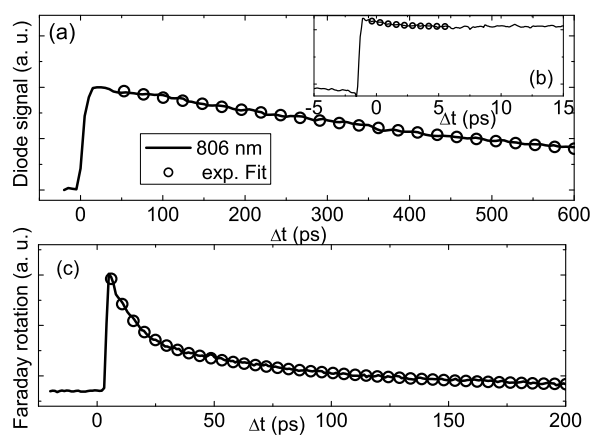

FIGURE 3. (a) Differential transmission over a broad time range. The inset (b) shows a measurement around $\Delta t=0$ with high temporal resolution. (c) Time resolved Faraday rotation.

we observe a biexponential decay of the TRFR signal, with decay constants $t_{1}=10 \mathrm{ps}$ and $t_{2}=81 \mathrm{ps}$, as deduced from Fig. 3c, which can be attributed to spin relaxation times of holes and electrons, respectively. These findings need an additional analysis, in particular regarding selective excitation of $X^{0}$ and $X^{+}$.

In summary, PL measurements show a significantly higher degree of spin polarization for trions than for neutral excitons. Time-resolved experiments indicate that for the neutral excitons, the photocarrier lifetime of hot excitons is longer than the spin relaxation time of the electrons. As a consequence, the majority of the neutral excitons loses its spin orientation during thermalization, before recombination. For the stronger polarization of the trions, two contributions may exist, which needs further investigation: Provided that the trions have a much shorter carrier lifetime than the neutral excitons, a larger part of them would decay radiatively before spin coherence is lost. In addition, the spin polarization of trions can be expected to be more stable because the two holes in the trion complex form the singlet spin state and the electronhole exchange is switched off and gives rise to no spin decoherence of the unpaired electron.

We acknowledge support by the Deutsche Forschungsgemeinschaft via SFB 689, GK 638 and project Schu1171/1.

\section{REFERENCES}

1. E.L. Ivchenko, Optical Spectroscopy of Semiconductor Nanostructures (Alpha Science, Harrow, 2005).

2. Gleb Finkelstein, Hadas Shtrikman, and Israel Bar-Joseph, Phys. Rev. B 53,R1709 (1995).

3. D. Sanvitto, R. A. Hogg, A. J. Shields, D. M. Whittaker, M. Y. Simmons, D. A. Ritchie, and M. Pepper, Phys. Rev. B 62, R13 294 (2000). 